\title{
Chemical Self-Assembly of Graphene Sheets
}

\author{
Hailiang Wang, Xinran Wang, Xiaolin Li, and Hongjie Dai $(\varangle)$ \\ Department of Chemistry and Laboratory for Advanced Materials, Stanford University, Stanford, CA 94305, USA \\ Received: 28 January 2009/Revised: 19 February 2009/ Accepted: 19 February 2009 \\ (C) Tsinghua University Press and Springer-Verlag 2009. This article is published with open access at Springerlink.com
}

\begin{abstract}
Chemically derived and noncovalently functionalized graphene sheets (GS) were found to self-assemble onto patterned gold structures via electrostatic interactions between the functional groups and the gold surfaces. This afforded regular arrays of single graphene sheets on large substrates, which were characterized by scanning electron microscopy (SEM), Auger microscopy imaging, and Raman spectroscopy. This represents the first time that self-assembly has been used to produce on-substrate and fully-suspended graphene electrical devices. Molecular coatings on the GS were removed by high current "electrical annealing", which restored the high electrical conductance and Dirac point of the GS. Molecular sensors for highly sensitive gas detection using the self-assembled GS devices are demonstrated.
\end{abstract}

\section{KEYWORDS}

Chemically derived graphene sheets, self-assembly, graphene devices, electrical annealing

Producing arrays of novel materials with ordered structures as device building blocks represents an important approach to nanoelectronics [14]. Graphene has recently been shown to have interesting properties including the quantum Hall effect [5], and potential applications in field effect transistors [6,7], sensors [8], and transparent electrodes [9]. Graphene was originally obtained by mechanical peeling-off [10] and epitaxial growth [11], but chemical methods [12-15] have recently attracted much attention due to their high yield and scalability. Thus far, graphene sheets (GS) made chemically have typically been deposited randomly on substrates. It is highly desirable to assemble GS at specific locations, and into desired patterns, for rational design of functional GS devices at a large scale. Only one report exists thus far of assembly of highly insulating graphite oxide (GO) [16] on metal surfaces.

Here, we report self-assembly of highlyconducting graphene sheets driven by electrostatic interactions between noncovalently attached functional groups on GS and gold surfaces. We found that GS functionalized by tetrabutylammonium (TBA) cations and phospholipid-polyethyleneglycolamine (PL-PEG-NH ${ }_{2}$ ) spontaneously adsorbed onto patterned gold surfaces on a silicon dioxide substrate. Auger elemental mapping/imaging, scanning electron microscopy (SEM), and Raman mapping revealed the high selectivity of GS deposition on gold rather than on the substrate. Arrays of single GS on substrates were obtained this way. By selfassembly, graphene sheets were directed to bridge arrays of gold electrodes on the substrate, affording large numbers of on-substrate GS devices and fully-

Address correspondence to hdai@stanford.edu 
suspended devices. An "electrical annealing" method was used to remove the coatings on GS, increase the electrical conductance of the GS device and make the GS dopant-free for applications such as chemical sensors.

Large quantities of high quality GS (sheet size $\sim 200 \mathrm{~nm}-1 \mu \mathrm{m}$ ) were made by an exfoliationreintercalation-expansion method [14]. In a typical making process (see Experimental section), ground natural graphite was intercalated by oleum in the presence of sodium nitrate, and then the product was treated by an aqueous solution of TBA hydroxide and suspended by PL-PEG-NH $\mathrm{N}_{2}$ in $\mathrm{N}, \mathrm{N}$-dimethylformamide (DMF) [17]. The final solvent had a volume ratio of $\mathrm{H}_{2} \mathrm{O}$ / DMF of $\sim 3 / 100$. Figure $1(b)$ is a schematic drawing of a GS functionalized noncovalently by TBA and PL-PEG-NH ${ }_{2}$, which forms a homogeneous and stable suspension in DMF. Such GS were shown previously to be highly conducting ( 100 fold more conducting than reduced graphene oxide) with a much lower degree of oxidation than commonly used graphite oxide [14]. An atomic force microscopy image (Fig. 1(a)) showed clean sheets of graphene on the silicon substrate. A height profile analysis (see

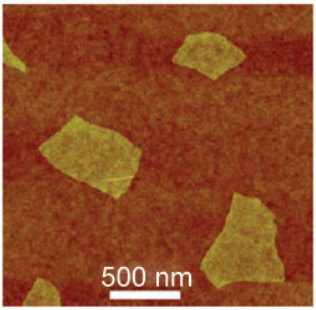

(a)

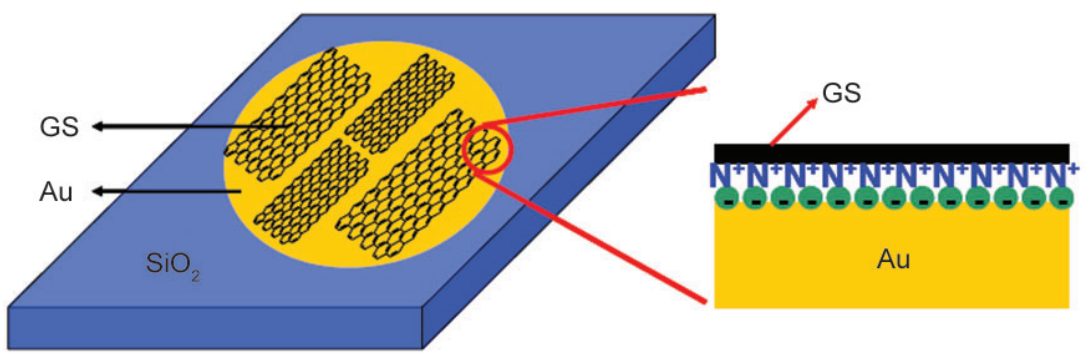

(c)

Figure 1 Self-assembly of graphene sheets (GS) on gold: (a) an AFM image of as-made GS; (b) a schematic drawing of noncovalently functionalized GS; (c) a schematic drawing of selective adsorption of GS on a gold pattern on silicon dioxide, mediated by electrostatic interactions between positively charged groups on GS and negative ions adsorbed on $\mathrm{Au}$ (right panel)
Electronic Supplementary Material (ESM), Fig. S-1) revealed that GS was $\sim 0.8-1 \mathrm{~nm}$ above the substrate, corresponding to single-layer graphene [12-14], as confirmed by the single symmetrical 2-D peak in the Raman spectrum of an individual GS (Fig. 21 and Fig. S-2(a) in the ESM).

Surface assembly of GS on gold occurred when we simply soaked silicon dioxide substrates with microfabricated gold patterns in a GS suspension, for 510 min (see Experimental section). Most of the GS were found to selectively adsorb onto gold surfaces, rather than onto the $\mathrm{SiO}_{2}$ substrate (Fig. 2). Scanning Auger elemental/chemical mapping and imaging were carried out to characterize the patterned GS structures. Carbon scanning Auger images (Figs. 2(b) and 2(e)) overlapped with those of gold (Figs. 2(c) and 2(f)), confirming selective self-assembly of GS onto the Au patterns.

To verify that we have achieved patterned assembly of single-layer GS, we carried out Raman mapping of GS assembled on gold arrays. The G-band Raman mapping image (Fig. 2(j)) clearly revealed assembled GS structures, consistent with the Auger mapping data. The high D/G (disorder to G band) ratio (Fig. 2(k)) can be attributed to the small sizes of GS $(\sim 400 \mathrm{~nm}$ average) compared to the laser spot $(\sim 1 \mu \mathrm{m})$, which probed defects on the edges of GS. Disorder could also be due to in-plane defects, but this was difficult to discern from the edges. The 2-D band of the GS was well fitted by a single Lorentzian peak, indicative of the single-layer nature $[18,19]$. In contrast, double-layer graphene showed a significantly different 2-D band with four superimposed peaks (see Fig. S-2(b) in the ESM).

By reducing the size of the gold patterns to $\sim 150 \mathrm{~nm}$, we pushed the limit of GS assembly on Au to the level of putting a single GS on a gold island. Figure 3(a) shows an SEM image of GS adsorbed onto small gold islands ( 150 $\mathrm{nm}$ size with $\sim 1.5 \mu \mathrm{m}$ pitch). Many of 


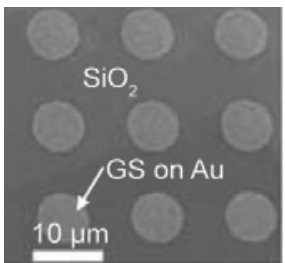

(a)

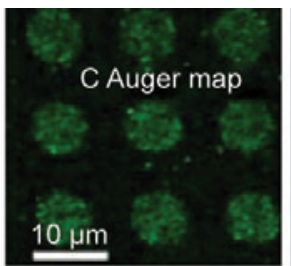

(b)

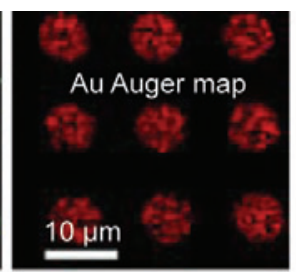

(c)

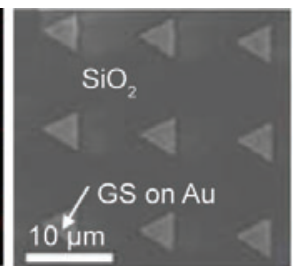

(d)

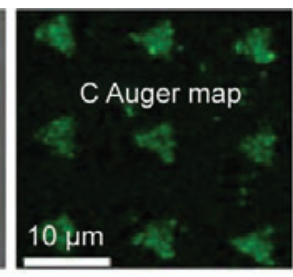

(e)

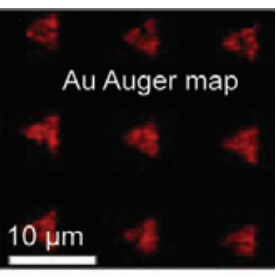

(f)

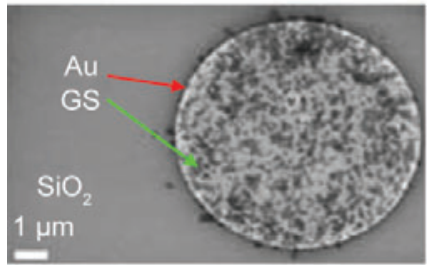

(g)

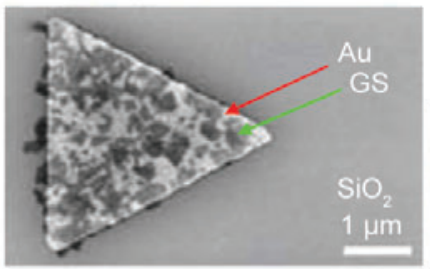

(h)

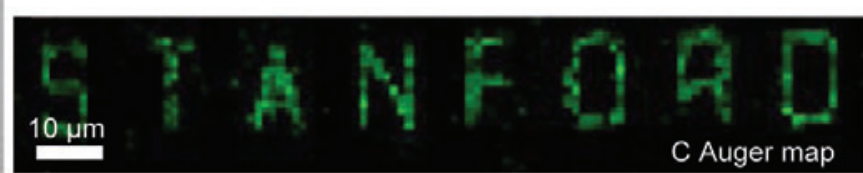

(i)

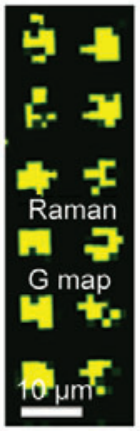

(j)

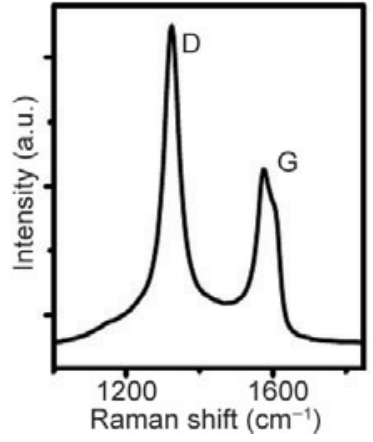

(k)

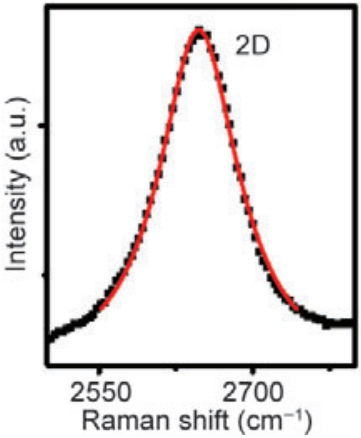

(I)

Figure 2 Self-assembled GS on two different patterns of gold structures on silicon dioxide, characterized by scanning electron microscopy, scanning Auger microscopy, and Raman spectroscopy. (a) and (d): SEM images. (b) and (c): Auger images for carbon and gold, respectively. (e) and (f): Auger images for carbon and gold, respectively. (g) and (h): SEM images showing GS adsorbed onto gold structures. The dark structures in the gold islands are self-adsorbed graphene sheets GS (sheet size $200 \mathrm{~nm}-1 \mu \mathrm{m}$ ). (i) Carbon scanning Auger image of the word "STANFORD" made of patterned Au lines with GS selectively adsorbed onto the word, i.e., graphene spells out "STANFORD". (j) Raman mapping (G-band) image of assembled GS arrays on Au. (k) and (I): Raman spectra of assembled GS on Au structures; in (I), the dotted line is the Raman 2-D band, and the red solid line is a single-Lorentzian fit

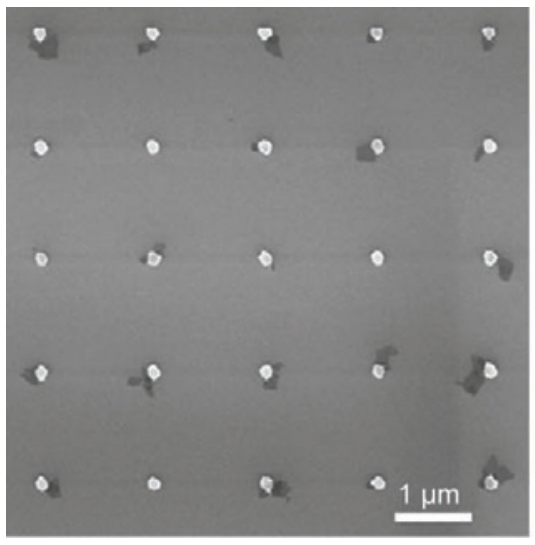

(a)

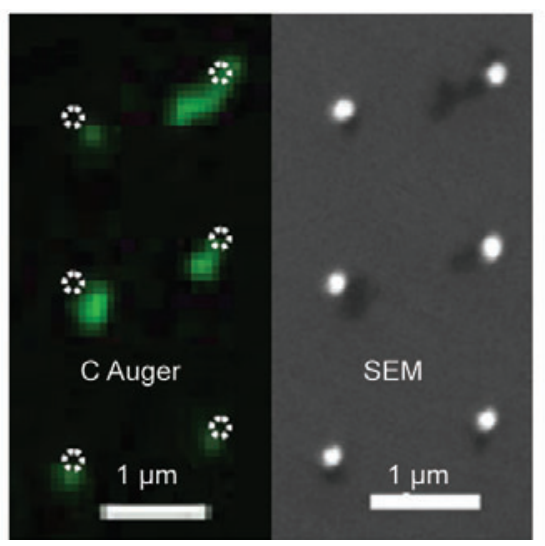

(b)

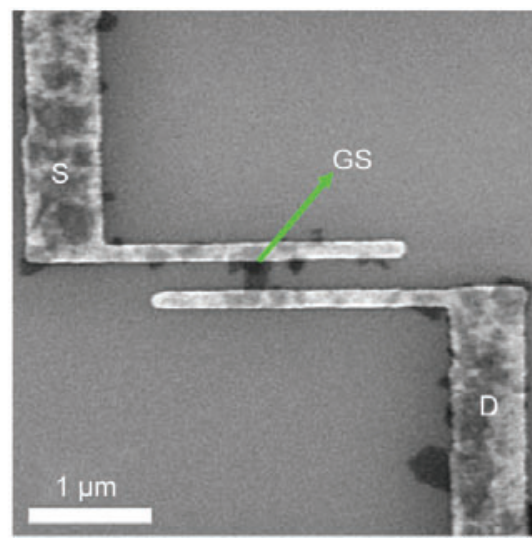

(c)

Figure 3 Single GS arrays and self-assembled GS devices: (a) an SEM image of small gold islands ( 150 nm) trapping 1 GS on each island. The dark structures attached to the gold islands are self-adsorbed GS; (b) side-by-side carbon scanning Auger image (left, white dotted circles are drawn to indicate the gold positions) and SEM image (right, dark structures are GS) of GS trapped on gold arrays; (c) an SEM image of GS adsorbed on gold electrodes with a single GS bridging two electrode fingers 
the Au islands have a single GS attached. Figure 3(b) shows a carbon scanning Auger image and an SEM image side by side. The C-Auger image of GS matched well with the SEM image of GS trapped on gold islands, showing Auger chemical imaging can be used to characterize single GS effectively.

Through a series of control experiments (see Table S-1 in the ESM), we investigated the underlying mechanism of GS self-assembly. Both TBA and the amino group of PL-PEG- $\mathrm{NH}_{2}$ can interact with the $\mathrm{Au}$ surface. However, our control experiments suggested that the presence of the latter was not critical. GS functionalized by TBA and PL-mPEG (with methyl terminal groups) showed similar selective adsorption on $\mathrm{Au}$ (see Fig. S-3(b) in the ESM), while GS with TBA removed by washing rarely adsorbed onto Au surfaces (see Fig. S-3(c) in the ESM). We also attempted assembly of GS on other metals including $\mathrm{Al}, \mathrm{Ti}, \mathrm{Co}$, and Pd. None of these metals showed as strong an attraction to GS as Au (see Fig. S-5 in the ESM). This is consistent with the strong electrostatic interactions between TBA and negatively-charged gold reported in Refs. [20, 21]. Our GS/DMF suspension contained $\sim 3 \%$ water by volume, which facilitated the electrostatic self-assembly process. No adsorption on Au could be observed if we transferred GS into pure DMF (see Fig. S-4(b) in the ESM). Hydroxide anions in the aqueous phase are known to adsorb onto Au surfaces to form a negatively charged layer $[22,23]$. These can then attract the positively charged GS/TBA for surface assembly. This was further verified by our control experiment in which we acidified the GS suspension with nitric acid (the $\mathrm{pH}$ was decreased from 9.5 to 4 ) to neutralize the hydroxide ions (nitrate anions are not known to adsorb strongly on $\mathrm{Au}$ ), and the adsorption of GS onto the Au surface was significantly reduced (see Fig. S-6 in the ESM).

The selective adsorption of GS on gold opens up a new way to build graphene devices. We soaked a substrate with preformed Au source-drain (S-D) electrode arrays in a GS suspension (see Experimental). The noncovalent self-assembly process navigated the TBA-functionalized GS onto the gold electrodes, with some of the GS bridging two closely spaced $\mathrm{Au}$ electrode fingers (Fig. 3(c)). This method affords an efficient way to fabricate GS electrical devices on a large scale. Typically, we made $\sim 100$ device patterns on a 0.5 $\mathrm{cm} \times 0.5 \mathrm{~cm}$ chip, and $10 \%-20 \%$ of them turned out to be single-connection GS devices.

Figure 4(a) shows an AFM image of a typical single GS device fabricated by the self-assembly method. The device was comprised of a $\mathrm{p}^{++}-\mathrm{Si}$ backgate, $100 \mathrm{~nm} \mathrm{SiO}_{2}$ as gate dielectric and $\mathrm{Ti}(2 \mathrm{~nm}) /$ $\mathrm{Au}(10 \mathrm{~nm})$ as source-drain electrodes (for bottom contacts). All fabricated GS devices were probed in a vacuum probe station with a base pressure of $\sim 2 \times 10^{-6}$ Torr. The as-made device showed almost no gate dependence with high resistance (Fig. 4(c), red curve). By sweeping to a high source drain bias of $\sim 4 \mathrm{~V}$, we observed that the current through the GS showed a sudden jump, with a large hysteresis upon sweeping back the bias voltage (Fig. 4(b)). The conductance of the device increased significantly (Fig. 4(c), blue curve) after this high current treatment cycle, which can be attributed to electrical annealing and heating effects on the GS and bottom contacts. It was previously reported that high-current annealing could heat up peeled-off graphene devices resulting in removal of contaminants and a shift of the Dirac point (DP) of graphene to near zero gate voltage [24]. Similar effects were observed with our chemically derived GS. After electrical annealing, a clear DP appeared around $V_{\mathrm{g}} \sim 0 \mathrm{~V}$, indicating very low doping level. Importantly, AFM imaging showed that the electrical annealing process removed coating molecules on the GS, with a $\sim 0.3-0.5 \mathrm{~nm}$ decrease in the apparent height or thickness of the GS after electrical annealing (see Fig. S-8 in the ESM). Thus, the electrical annealing step clearly removed the molecules on the GS responsible for the self-assembly process and the associated doping effect, leading to graphene sheet devices exhibiting the DP that is intrinsic to pristine graphene.

We also obtained fully-suspended graphene sheet devices by self-assembly of GS between tall metal electrodes. We shortened the source-drain electrode distance to less than $100 \mathrm{~nm}$ and increased the electrode height to $\sim 50 \mathrm{~nm}$. Graphene sheets were able to adsorb and bridge the electrodes via self-assembly and became suspended. This simple solution processing method readily leads to 
suspended GS devices (Fig. 4(d)) exhibiting a DP after electrical annealing (Fig. 4(f)) without using sophisticated fabrication/etching methods [25-27].

Our GS was made by a mild process without extensive covalent functionalization of the GS [14] and electrical annealing was used to remove the molecular coating and increase the conductance of the GS devices. The average resistivity (defined as $\frac{R \times W}{L}$, where $R$ is the resistance of the device, and $W$ and $L$ indicate GS width and channel length, respectively) at the minimum conductance point (MCP) was $\sim 30 \mathrm{k} \Omega$ after electrical annealing (Fig. $4(\mathrm{~g})$ ), about 100 times lower than commonly used GO in its reduced state $[12,13,15]$. Our GS exhibited $\sim 2-5$ times higher resistivity than pristine peel-off graphene [24, 28] (Fig. 4(g)), suggesting small amount of disorder in the plane of the GS, which could also contribute to the D peak observed by Raman

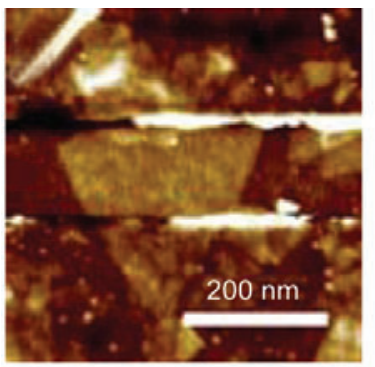

(a)

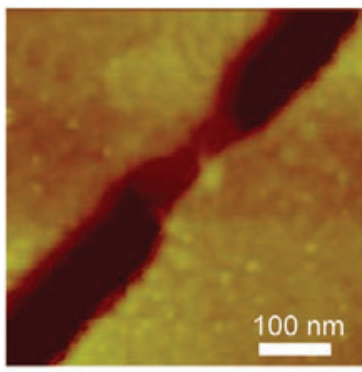

(d)

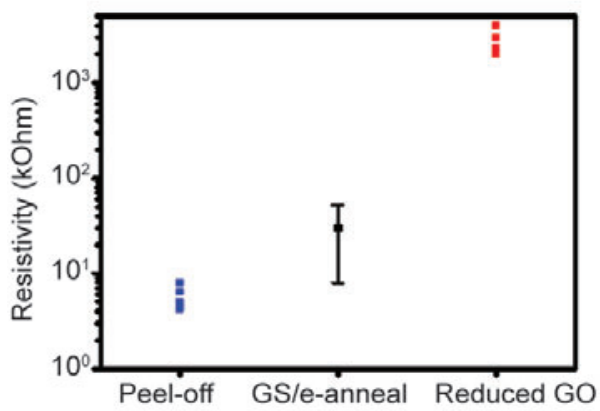

(g)

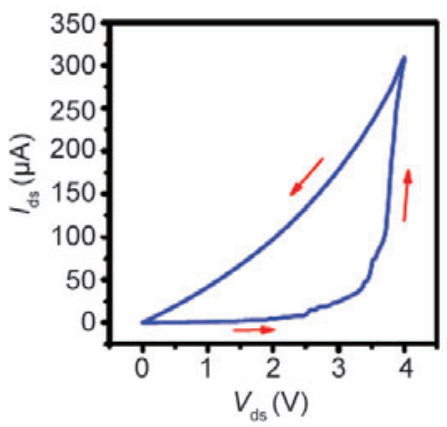

(b)

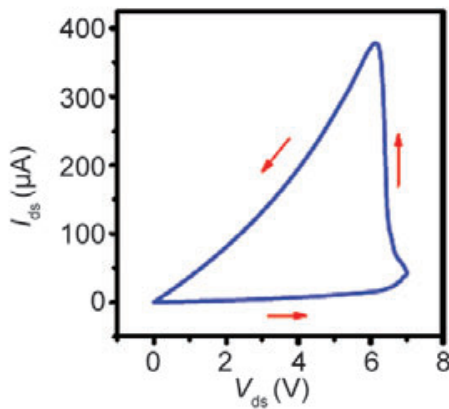

(e)

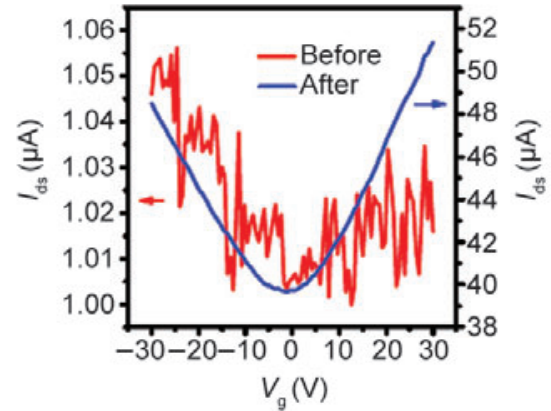

(c)

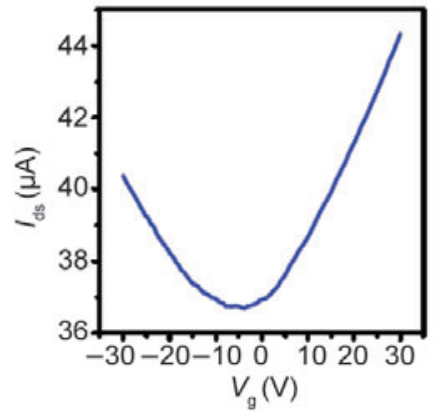

(f)

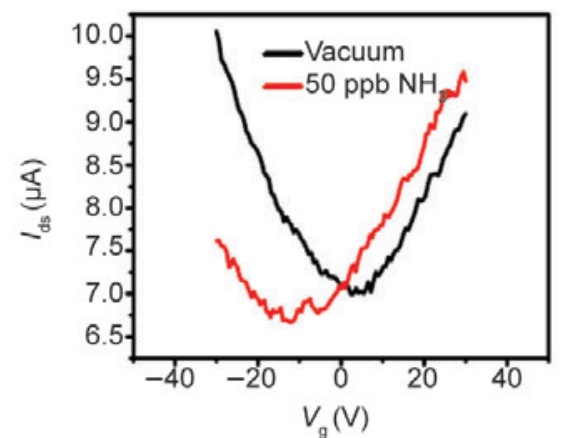

(h)

Figure 4 Electrical devices composed of self-assembled graphene on-substrate and in fully suspended form. (a) An AFM image of a single GS bridging two Au electrodes on a substrate. (b) Electrical annealing current-voltage curves recorded by sweeping the bias voltage across the GS. The red arrows show the direction of the voltage sweep. (c) Current-gate voltage curves $\left(V_{\mathrm{ds}}=1 \mathrm{~V}\right)$ of the same GS device before and after electrical annealing. (d) An AFM image of a suspended GS device. (e) Electrical annealing current-voltage curves recorded by sweeping the bias voltage across the GS in (d). ( $f$ ) Current-gate voltage curves $\left(V_{\mathrm{ds}}=1 \mathrm{~V}\right)$ of the same $\mathrm{GS}$ device before and after electrical annealing. ( $\mathrm{g}$ ) Resistivity comparison of different graphene devices. The resistivity value of electrically annealed GS devices was the average of more than 30 devices, with the error bar indicating the standard deviation. The multiple resistivity values of peel-off graphene and reduced GO are based on electrical transport data in the literature (see ESM). (h) Current-gate voltage curves $\left(V_{\mathrm{ds}}=1 \mathrm{~V}\right.$ ) of a single $\mathrm{GS}$ device before and after exposure to $50 \mathrm{ppb}$ ammonia gas 
spectroscopy. Nevertheless, our GS devices are 100 times more conducting than reduced GO with a clear DP similar to pristine graphene. Such devices are useful for various applications, as demonstrated by ammonia detection at the ppb level (Fig. 4(h)) $[8,29,30]$. Ammonia caused a clear shift in the DP of our GS as a result of n-doping, which serves as the basis for molecular detection unattainable with graphene devices without Dirac points and little gate-dependence of the electrical conductance.

\section{Experimental}

Making of GS was developed from our previous work [14]. Typically, $20 \mathrm{mg}$ natural graphite flakes were ground with $\mathrm{NaCl}$ crystallites. After removing $\mathrm{NaCl}$ by washing with water and filtration, the resulting solid was soaked in $10 \mathrm{~mL}$ oleum (with $20 \%$ free $\mathrm{SO}_{3}$ ) and $1 \mathrm{~g} \mathrm{NaNO}_{3}$, and stirred for 1 day. The acid and salt were removed by washingrepeatedly and thoroughly with water, and the solid obtained was then dispersed in $10 \mathrm{~mL} \mathrm{DMF}$, to which $300 \mu \mathrm{L}$ of an aqueous solution (40\%) of TBA hydroxide was added. After $10 \mathrm{~min}$ of cup-horn sonication, the suspension was kept still for 2 days, and then a $4 \mathrm{~mL}$ aliquot was removed and bath-sonicated with $15 \mathrm{mg}$ phospholipid-polyethyleneglycol-amine (PL-PEG$\mathrm{NH}_{2}, \mathrm{MW} 5000$ ) for $1 \mathrm{~h}$, forming a homogeneous suspension. After centrifuging (15000 rpm) for $3 \mathrm{~min}$, a black homogeneous supernatant was obtained.

$\mathrm{Au}$ patterns on $\mathrm{SiO}_{2}$-coated $(\sim 100 \mathrm{~nm}) \mathrm{Si}$ substrates were fabricated through a procedure of electron beam lithography (EBL), metallization, and lift-off. The patterned substrate was soaked in a GS suspension for 5-10 min, and then rinsed with water and 2-propanol, and finally dried in an argon flow.

The GS devices were obtained in similar way. A typical on-substrate device was comprised of a $\mathrm{p}^{++}$Si backgate, $100 \mathrm{~nm} \mathrm{SiO}_{2}$ as gate dielectric and $\mathrm{Ti}(2$ $\mathrm{nm}) / \mathrm{Au}(10 \mathrm{~nm})$ as source-drain electrodes. $\mathrm{Pd}(40$ $\mathrm{nm}) / \mathrm{Au}(10 \mathrm{~nm})$ were used as source-drain electrodes for fully suspended GS device fabrication. After electrode fabrication, the chip was soaked in a GS suspension for $\sim 5 \mathrm{~min}$, and then rinsed by water and 2-propanol, and finally dried in an argon flow. All fabricated GS devices were probed in a vacuum probe station with a base pressure of $\sim 2 \times 10^{-6}$ Torr.

AFM images of the GS were taken with a Nanoscope IIIa multimode instrument. Scanning Auger elemental mapping was carried out with $\mathrm{PHI} 700^{\mathrm{TM}}$ scanning Auger nanoprobe. The mapping images were plotted using the $\sim 275 \mathrm{eV}$ (KLL) peak for carbon, and the $\sim 75$ $\mathrm{eV}(\mathrm{MNN})$ peak for gold. Raman mapping images and spectra were taken with HORIBA Jobin Yvon Raman spectrometer (633 nm laser excitation). SEM images were obtained using a Raith 150 nanofabrication system. Electrical characteristics of fabricated devices were measured with Hewlett Packard 4156B precision semiconductor parameter analyzer.

\section{Acknowledgements}

This work is supported by MARCO-MSD, ONR, and Intel.

Electronic Supplementary Material: Further characterization of GS and self-assembled structures by AFM, Raman, and Auger can be found in the ESM with nine figures and one table which are available in the online version of this article at http://dx.doi. org/10.1007/s12274-009-9031-x and accessible free of charge.

\section{References}

[1] Fan, S. S.; Chapline, M. G.; Franklin, N. R.; Tombler, T. W.; Cassell, A. M.; Dai, H. J. Self-oriented regular arrays of carbon nanotubes and their field emission properties. Science 1999, 283, 512-514.

[2] Huang, M. H.; Mao, S.; Feick, H.; Yan, H. Q.; Wu, Y. Y.; Kind, H.; Weber, E.; Russo, R.; Yang, P. D. Roomtemperature ultraviolet nanowire nanolasers. Science 2001, 292, 1897-1899.

[3] Liu, Y. L.; Li, H. X.; Tu, D. Y.; Ji, Z. Y.; Wang, C. S.; Tang, Q. X.; Liu, M.; Hu, W. P.; Liu, Y. Q.; Zhu, D. B. Controlling the growth of single crystalline nanoribbons of copper tetracyanoquinodimethane for the fabrication of devices and device arrays. J. Am. Chem. Soc. 2006, 128, 1291712922.

[4] Dai, H. Carbon nanotubes: synthesis, integration, and properties. Acc. Chem. Res. 2002, 35, 1035-1044.

[5] Zhang, Y. B.; Tan, Y. W.; Stormer, H. L.; Kim, P. 
Experimental observation of the quantum Hall effect and Berry's phase in graphene. Nature 2005, 438, 201-204.

[6] Li, X. L.; Wang, X. R.; Zhang, L.; Lee, S. W.; Dai, H. J. Chemically derived, ultrasmooth graphene nanoribbon semiconductors. Science 2008, 319, 1229-1232.

[7] Wang, X. R.; Ouyang, Y. J.; Li, X. L.; Wang, H. L.; Guo, J.; Dai, H. J. Room-temperature all-semiconducting sub-10$\mathrm{nm}$ graphene nanoribbon field-effect transistors. Phys. Rev. Lett. 2008, 100, 206803.

[8] Schedin, F.; Geim, A. K.; Morozov, S. V.; Hill, E. W.; Blake, P.; Katsnelson, M. I.; Novoselov, K. S. Detection of individual gas molecules adsorbed on graphene. Nat. Mater. 2007, 6, 652-655.

[9] Wang, X.; Zhi, L. J.; Müllen, K. Transparent, conductive graphene electrodes for dye-sensitized solar cells. Nano Lett. 2008, 8, 323-327.

[10] Novoselov, K. S.; Geim, A. K.; Morozov, S. V.; Jiang, D.; Zhang, Y.; Dubonos, S. V.; Grigorieva, I. V.; Firsov, A. A. Electric field effect in atomically thin carbon films. Science 2004, 306, 666-669.

[11] Sutter, P. W.; Flege, J; Sutter, E. A. Epitaxial graphene on ruthenium. Nat. Mater. 2008, 7, 406-411.

[12] Li, D.; Müller, M. B.; Gilje, S.; Kaner, R. B.; Wallace, G. G. Processable aqueous dispersions of graphene nanosheets. Nat. Nanotechnol. 2008, 3, 101-105.

[13] Gomez-Navarro, C.; Weitz, R. T.; Bittner, A. M.; Scolari, M.; Mews, A.; Burghard, M.; Kern, K. Electronic transport properties of individual chemically reduced graphene xxide sheets. Nano Lett. 2007, 7, 3499-3503.

[14] Li, X. L.; Zhang, G. Y.; Bai, X. D.; Sun, X. M; Wang, X. R.; Wang, E. G.; Dai, H. J. Highly conducting graphene sheets and Langmuir-Blodgett films. Nat. Nanotechnol. 2008, 3, 538-542.

[15] Stankovich, S.; Dikin, D. A.; Piner, R. D.; Kohlhaas, K. A.; Kleinhammes, A.; Jia, Y. Y.; Wu, Y.; Nguyen, S. T.; Ruoff, R. S. Synthesis of graphene-based nanosheets via chemical reduction of exfoliated graphite oxide. Carbon 2007, 45, 1558-1565.

[16] Wei, Z. Q.; Barlow, D. E.; Sheehan, P. E. The assembly of single-layer graphene oxide and graphene using molecular templates. Nano Lett. 2008, 8, 3141-3145.

[17] Liu, Z. H.; Wang, Z. M.; Yang, X. J.; Ooi, K. Intercalation of organic ammonium ions into layered graphite oxide. Langmuir 2002, 18, 4926-4932.

[18] Ferrari, A. C.; Meyer, J. C.; Scardaci, V.; Casiraghi, C.; Lazzeri, M.; Mauri, F.; Piscanec, S.; Jiang, D.; Novoselov,
K. S.; Roth, S.; Geim, A. K. Raman spectrum of graphene and graphene layers. Phys. Rev. Lett. 2006, 97, 187401.

[19] Eda, G.; Fanchini, G.; Chhowalla, M. Large-area ultrathin films of reduced graphene oxide as a transparent and flexible electronic material. Nat. Nanotechnol. 2008, 3, 270-274.

[20] Chen, S. M.; Liu, Y. D.; Wu, G. Z. Stabilized and size-tunable gold nanoparticles formed in a quaternary ammoniumbased room-temperature ionic liquid under $\gamma$-irradiation Nanotechnology 2005, 16, 2360-2364.

[21] Tymosiak-Zieliska, A.; Borkowska, Z. Interfacial properties of polycrystalline gold electrodes in tetraalkylammonium electrolytes. Electrochim. Acta 2001, 46, 3073-3082.

[22] Biggs, S.; Mulvaney, P.; Zukoski, C. F.; Grieser, F. Study of anion adsorption at the gold-aqueous solution interface by atomic force microscopy. J. Am. Chem. Soc. 1994, 116, 9150-9157.

[23] Bockris, J. O'M.; Paik, W. K.; Genshaw, M. A. Adsorption of anions at the solid-solution interface. Ellipsometric study J. Phys. Chem. 1970, 74, 4266-4275.

[24] Moser, J.; Barreiro, A.; Bachtold, A. Current-induced cleaning of graphene. Appl. Phys. Lett. 2007, 91, 163513.

[25] Garcia-Sanchez, D.; van der Zande, A. M.; San Paulo, A.; Lassagne, B.; McEuen, P. L.; Bachtold, A. Imaging mechanical vibrations in suspended graphene sheets. Nano Lett. 2008, 8, 1399-1403.

[26] Gómez-Navarro, C.; Burghard, M.; Kern, K. Elastic properties of chemically derived single graphene sheets. Nano Lett. 2008, 8, 2045-2049.

[27] Bunch, J. S.; van der Zande, A. M.; Verbridge, S. S.; Frank, I. W.; Tanenbaum, D. M.; Parpia, J. M.; Craighead, H. G.; McEuen, P. L. Electromechanical resonators from graphene sheets. Science 2007, 315, 490-493.

[28] Miao, F.; Wijeratne, S.; Zhang, Y.; Coskun, U.; Bao, W.; Lau, C. N. Phase-coherent transport in graphene quantum billiards. Science 2007, 317, 1530-1533.

[29] Kong, J.; Franklin, N.; Zhou, C. W.; Chapline, M. G.; Pan, S.; Cho, K. J.; Dai, H. J. Nanotube molecular wires as chemical sensors. Science 2000, 287, 622-625.

[30] Chen, R. J.; Bangsaruntip, S.; Drouvalakis, K. A.; Kam, N. W. S.; Shim, M.; Li, Y. M.; Kim, W.; Utz, P. J.; Dai, H. J. Noncovalent functionalization of carbon nanotubes for highly specific electronic biosensors. Proc. Natl. Acad. Sci. USA. 2003, 100, 4984-4989. 